\title{
EchoGéo
}

$14 \mid 2010$

Afrique, 50 ans d'indépendance : Dynamiques spatiales, identités, circulations

\section{Les nouveaux programmes scolaires de géographie au Lycée \\ Introduction}

Alexis Sierra

\section{OpenEdition \\ Journals}

Édition électronique

URL : https://journals.openedition.org/echogeo/12225

DOI : $10.4000 /$ echogeo. 12225

ISSN : 1963-1197

\section{Éditeur}

Pôle de recherche pour l'organisation et la diffusion de l'information géographique (CNRS UMR 8586)

\section{Référence électronique}

Alexis Sierra, "Les nouveaux programmes scolaires de géographie au Lycée », EchoGéo [En ligne], 14 | 2010, mis en ligne le 16 décembre 2010, consulté le 31 juillet 2021. URL : http://

journals.openedition.org/echogeo/12225 ; DOI : https://doi.org/10.4000/echogeo.12225

Ce document a été généré automatiquement le 31 juillet 2021.

EchoGéo est mis à disposition selon les termes de la licence Creative Commons Attribution - Pas d'Utilisation Commerciale - Pas de Modification 4.0 International (CC BY-NC-ND) 


\section{Les nouveaux programmes scolaires de géographie au Lycée}

Introduction

Alexis Sierra

1 La publication des nouveaux programmes de géographie de la classe de Seconde du lycée est l'occasion de faire le point sur ce que représentent des programmes scolaires pour l'évolution de notre discipline. Ceux-ci sont en effet le produit des adaptations à la recherche scientifique, des préoccupations politiques et sociales, des évolutions institutionnelles et pédagogiques. Concernant le contexte politique, les programmes ont pu être déterminés aussi bien par des changements de régime que par des contraintes budgétaires ou par la volonté de la part d'un gouvernement d'imprimer sa marque ou de réformer l'Etat. C'est ainsi que les programmes de géographie de lycée ont connu une quinzaine de versions appliquées entre le second Empire et cette année 2010. Toutes ne sont pas marquées du sceau particulier d'un ministre comme sous Jean Zay, mais elles sont souvent liées aux changements de gouvernement.

Chronologie des programmes scolaires en géographie

\begin{tabular}{|l|l|}
\hline 1865 & Victor Duruy ; Seconde Empire \\
\hline $1872-1874$ & Débuts de la III ${ }^{\mathrm{e}}$ République ; commission Emile Levasseur \\
\hline 1890 & Léon Bourgeois ; Républicains \\
\hline 1902 & Radicaux \\
\hline 1905 & Radicaux \\
\hline 1925 & \\
\hline 1938 & Jean Zay ; Front Populaire \\
\hline
\end{tabular}




\begin{tabular}{|l|l|}
\hline 1942 & Jérôme Carcopino ; régime de Vichy \\
\hline 1945 & René Capitant ; gouvernement de la Libération \\
\hline 1957 & René Billères \\
\hline 1978 & René Haby \\
\hline $1986-1990$ & Jean-Pierre Chevènement ; actualisation Lionel Jospin \\
\hline 1995 & François Bayrou \\
\hline 2001 & Jack Lang \\
\hline
\end{tabular}

Colonne de droite : le ministre en charge de l'Education (source INRP) et éventuellement le régime.

Source : Audigier, 2001 ; Wastable, 2010.

2 La refonte actuelle fait suite à une réforme plus large du système éducatif dans un contexte de restriction des dépenses publiques. Elle intervient après la réforme du lycée et à la suite de la réécriture des programmes du collège. La géographie scolaire (comme l'histoire) est en particulier touchée par la suppression de son enseignement obligatoire en Terminale S. C'est un changement majeur voire régressif si on considère que la géographie avait été introduite en terminale en 1905. Chaque représentant d'une discipline tend à la défendre. Il n'est cependant pas corporatiste de s'interroger sur les conséquences de cette suppression d'obligation alors que le monde se conçoit comme plus complexe et que tout citoyen - plus encore tout travailleur - est appelé à davantage de mobilité. Rappelons que l'enseignement de la géographie fut renforcé à la suite de la guerre de 1870 pour précisément éviter de nouvelles défaites et que la géographie scolaire est un gage de démocratie pour une discipline réservée aux autorités publiques, civiles et militaires, dans les pays autoritaires.

3 Les réformes de l'Etat et de l'enseignement ont donc conduit le gouvernement français à rédiger de nouveaux programmes scolaires notamment pour la géographie. La classe de Seconde restant en théorie indéterminée, ce n'est pas à ce niveau que les changements doivent être les plus profonds. On pourra d'ailleurs constater une relative continuité entre les programmes appliqués depuis 2001 et les nouveaux.

Présentation comparative des programmes de géographie en Seconde en 2001 et 2010

\begin{tabular}{|l||l|}
\hline Programme de Seconde de 2001 & Programme de Seconde de 2010 \\
\hline $\begin{array}{l}\text { Thème introductif - Plus de six } \\
\text { milliards d'hommes sur la Terre }\end{array}$ & $\begin{array}{l}\text { Thème introductif - Les enjeux du développement : } \\
\text { du développement au développement durable }\end{array}$ \\
\hline $\begin{array}{l}\text { - L'inégale répartition des hommes et } \\
\text { des richesses sur la terre }\end{array}$ & $\begin{array}{l}\text { - Un développement inégal et déséquilibré à toutes les } \\
\text { échelles }\end{array}$ \\
\hline $\begin{array}{l}\text { - Une division entre États, mais des } \\
\text { enjeux transnationaux }\end{array}$ & $\begin{array}{l}\text { - De nouveaux besoins pour plus de 9 milliards d'hommes } \\
\text { en } 2050\end{array}$ \\
\hline
\end{tabular}




\begin{tabular}{|c|c|}
\hline $\begin{array}{l}\text { - Frontières, aménagements et } \\
\text { environnement }\end{array}$ & - Mettre en œuvre des modes durables de développement \\
\hline Autres thèmes obligatoires & $\begin{array}{l}\text { Thème } 2 \text { - Gérer les ressources terrestres (deux } \\
\text { questions aux choix parmi les trois proposées) }\end{array}$ \\
\hline Nourrir les hommes & Nourrir les hommes \\
\hline L'eau, entre abondance et rareté & L'eau, ressource essentielle \\
\hline Les sociétés face aux risques & $\begin{array}{l}\text { L'enjeu énergétique - Besoin en énergie et gestion des } \\
\text { ressources. }\end{array}$ \\
\hline \multirow[t]{4}{*}{$\begin{array}{l}\text { Dynamiques urbaines et } \\
\text { environnement urbain }\end{array}$} & $\begin{array}{l}\text { Thème } 3 \text { - Aménager la ville - Villes et } \\
\text { développement durable (Question obligatoire) }\end{array}$ \\
\hline & $\begin{array}{l}\text { - Croissance urbaine, étalement urbain, inégalités socio- } \\
\text { spatiales }\end{array}$ \\
\hline & - Transports et mobilités \\
\hline & - Aménager des villes « durables»? \\
\hline Un thème au choix & $\begin{array}{l}\text { Thème } 4 \text { - Gérer les espaces terrestres (deux questions } \\
\text { aux choix parmi les trois proposées) }\end{array}$ \\
\hline Les littoraux, espaces attractifs & $\begin{array}{l}\text { Les mondes arctiques, une «nouvelle frontière " sur la } \\
\text { planète }\end{array}$ \\
\hline \multirow[t]{2}{*}{$\begin{array}{l}\text { Les montagnes, entre traditions et } \\
\text { nouveaux usages }\end{array}$} & Les littoraux, espaces convoités \\
\hline & Les espaces exposés aux risques majeurs \\
\hline
\end{tabular}

\section{Source : Ministère de l'Education Nationale.}

4 Il sera sans doute intéressant de réaliser ce comparatif à l'occasion de la publication des nouveaux programmes de Première puisque pour la filière « $S$ », la Première sera la dernière année de géographie obligatoire.

5 Cependant, la comparaison des programmes de Seconde est éclairante quant à l'évolution de la géographie scolaire et soulève des questions par rapport à ce qu'est la géographie.

6 La relation de la géographie à la notion de développement durable est extrêmement présente. Nous y reviendrons. Le programme de 2001 insistait en introduction sur la géographie de la population et sur l'organisation politique des Hommes sur la Terre. Avec une réflexion autour du découpage en Etats, autour des espaces frontaliers ainsi que des espaces transfrontaliers et des enjeux transnationaux, Le premier chapitre avait une évidente perspective géopolitique. Le programme de 2010 part, quant à lui, de la relation aux ressources en relation avec leurs limites et avec les inégalités de développement; ce qui vaut aussi bien pour le thème introductif que pour les deux 
chapitres suivant à enseigner. Le devenir de l'humanité qu'intègre le terme " durable " se traduit par le titre du paragraphe touchant au peuplement de la Terre : il ne s'agit plus de décrire la situation des 6 milliards d'habitants comme en 2001 mais d'avoir une vision prospective à l'horizon de 9 milliards d'habitants en 2050.

7 L'enseignement des territoires urbains est renforcé par rapport au précédent programme. La représentation de la ville à la fois comme moteur, foyer et laboratoire du développement de l'espace mondial n'est sans doute pas étrangère à cette orientation. Ce qui peut surprendre, c'est que l'urbain n'est pas traité comme une entrée du thème quatre, alors qu'il s'agit d'un type d'espace. Inversement, le risque qui aurait pu être transversal à plusieurs espaces est renvoyé à ce thème 4 défini par des entrées spatiales. Le risque pouvait être en effet une grille de lecture pour traiter plusieurs chapitres (l'eau, des inondations aux sécheresses en passant par les pollutions ; l'alimentation avec la sécurité alimentaire ; l'énergie avec les risques liées à la production, le transport et la consommation d'énergie, etc...). Si le risque a une spatialisation, les espaces soumis aux risques couvrent la planète entière. Cela rend la définition du cours difficile et diffère des autres items proposés dans ce thème 4 : l'arctique et les littoraux.

8 La forte articulation de la géographie au développement durable donne l'impression d'un effet de mode. Elle est cependant l'occasion de penser la complexité du monde et d'articuler les différentes échelles, en phase également avec l'étude de la mondialisation. Enfin, cela peut être l'occasion de renouveler la représentation de la géographie physique en relation étroite avec les préoccupations sociales.

Pour avoir un éclairage sur l'élaboration de ce nouveau programme et des enjeux, nous avons fait appel à François Louveau, Inspecteur Général de l'Education Nationale et Philippe Sierra, professeur en lycée. Ces deux géographes portent deux visions qui parfois se retrouvent et d'autres fois divergent sensiblement. Le premier est contraint par sa position de représentant du ministère. Même s'il n'a pas été mandaté pour être porte parole de l'Institution, il en traduit la politique. Son souci est de mettre en application ces programmes et non de discuter des choix opérés même s'il nous aide à comprendre les choix réalisés. Inversement, le second s'exprime du point de vue du praticien au quotidien qui doit appliquer les programmes : il n'appartient pas à ceux qui ont choisi et il en discute certains choix.

10 C'est ainsi que les points de vues se différencient sensiblement quant aux conditions d'élaboration des programmes et à sa signification institutionnelle. Cependant, nos deux interlocuteurs se retrouvent dans la portée pédagogique des changements: mettre en place un enseignement de la géographie qui sache être en phase avec les préoccupations contemporaines et puisse répondre aux interrogations des élèves. Tous deux reconnaissent les éléments de continuité et la nécessité de trouver des vecteurs pour intéresser les élèves à la géographie. La formation et les ressources documentaires font partie de leurs préoccupations face à la notion de développement durable.

11 Nous espérons que ces deux entretiens donneront quelques pistes sur le rôle de la géographie et sur les enjeux de la géographie scolaire. Si besoin était, rappelons que le recrutement des étudiants en géographie dépend de ce qui a été fait dans le secondaire. Rappelons aussi que l'inscription de thèmes au programme des concours de recrutement induit des publications, des conférences et stimule donc la recherche et les travaux réflexifs. 


\section{AUTEUR}

\section{ALEXIS SIERRA}

Alexis Sierra est maître de conférences en géographie à l'université Cergy-Pontoise-IUFM. alexisierra2001@yahoo.fr 